\title{
Joint loading estimation method for horse forelimb high jerk locomotion: jumping
}

\author{
Becker Joanne $^{1-2}$, Mermoz Emmanuel ${ }^{1-2}$, Linares Jean-Marc ${ }^{2}$ \\ 1. Airbus Helicopters, Aéroport de Marseille Provence, 13700 Marignane, France \\ 2. Aix Marseille Univ, CNRS, ISM, Inst Movement Sci, 13009 Marseille, France
}

Corresponding author email: joanne.becker@univ-amu.fr

\begin{abstract}
Maximal local loads in animal joints are necessary to design bio-inspired mechanical joints. Many studies presented methods to determine joint reaction forces in humans and animals. However, many of these methods are invasive, and no work has been published yet about the joint reaction forces in the horse forelimb during jumping. Non-invasive methods to measure the kinematics and ground reaction force of a horse forelimb were used in this work. A musculoskeletal model of horse forelimb was built with mechanical methods for the estimation of joint reaction forces. The entire forelimb was reconstructed by scanning real bones geometry with a 3D optical scanner and modeling all the muscles on a Computer Assisted Design (CAD) software. The model dynamics were simulated with OpenSim in order to estimate the joint loading. This study allows knowing an order of magnitude of the loads at the joints at jumping in order to determine latter the maximal joint contact loading values that will be a key at designing bio-inspired joints for mechanical assemblies.
\end{abstract}

Keywords: joint loading, jumping, kinematics, modelling, OpenSim

\section{Introduction}

The imitation of highly performing articulations could lead to a crucial change in the design of current mechanical systems. Even if animal joints areusually simplified as hinge or spherical joints ${ }^{[1]}$, their articulations present complex contact surfaces that should take part in locomotion performances.Unguligrade animals show interesting locomotion characteristics because their vertebral column is stable and their locomotor limbs move principally in sagittal plane. Horses in particular are real athletes, presenting high sportive performances and large loads in their joints ${ }^{[2]}$. The horse was chosen as the experimental subject of this study because it is easily accessible and very easy to train.

In order to mimick these articulations, it is necessary to know the local pressures through these joints. In Picault et al. (2018) ${ }^{[3]}$, the authors gave a method to determine the contact areas and local pressure values with finite elements. These local pressures cannot be computed without the determination of joint loadings of an animal articulation. The aim of our work is therefore to determine the equivalent joint loadings of horse forelimb articulations for a high jerk in order to compute latter the local pressures to design bio-inspired joints.

Several works in medical sciences have investigated the estimation of joint reaction forces in humans. On the one hand, this enables to assess the characteristics of reactions in joints (force, direction...), and on the other hand, to understand injuries, fatigue fractures... In human, many athletes and aged people present hip injuries ${ }^{[4-5]}$, and knee injuries ${ }^{[6-7]}$. The joint reaction forces information would enable to better design knee or hip prostheses in order to improve their lifetime and to reduce the pains. In humans, these estimations can be assessed by in vivo measurements using the prostheses as measuring devices 
${ }^{[5]}$, but this method is invasive and costly. In the recent years, researchers have developed modelling techniques for a better understanding of movement dynamics and estimation of internal joint reaction forces. One of these methodologies is the finite element modelling which was widely used to better understand joint behavior in human ${ }^{[8-10]}$. Another developed method is the musculoskeletal modelling which first has been applied on human lower limb modelling ${ }^{[11-14]}$ but more recently also on animals like horse $^{[2,15]}$, sheeps ${ }^{[1]}$ and ostriches ${ }^{[16]}$.

The work presented in this paper aims at building a musculoskeletal model of the entire forelimb of the horse to compute the joint reactions forces at jumping. For this purpose, this work first presents noninvasive measurements to collect markers trajectories and ground reaction forces values. Then the methods for the modelling of the complete horse forelimb and for the computation of the joint reaction forces are presented. The joint loadings were first computed at trotting to compare with literature and then at high jerk locomotion by jumping a fence of $1 \mathrm{~m}$ high.

\section{Materials and methods}

\subsection{Animal used for in vivo experiments}

For the experiments, a French Saddle sport horse weighing $560 \mathrm{~kg}$ and judged free of obvious lameness was ridden by a professional rider. The total weight of the horse, his rider and the equipment was about $650 \mathrm{~kg}$.

\subsection{Gait instrumentation}

In these experiments, the video and force plate data were simultaneously recorded for the horse and his rider for both trotting and jumping a small fence of $1 \mathrm{~m}$ high. Seven non-distracting principal markers were attached to the animal's skin: top of scapula, shoulder, elbow, wrist joint, metacarpophalangeal (MCP) joint, phalangeal (P1-P2) joint, extremity of the hoof (Fig. 1). Four secondary markers were placed on the long segments to determine the position and orientation of each segment during gait. No secondary markers were placed on the phalanxes. Too many markers on such short segments could lead to some confusions when following their trajectories. A $60 \mathrm{~Hz} 1440 \mathrm{p}$ board camera was following the horse, at a distance $(d)$, in order to build a travelling system and to simplify mathematical processing like distortion (Fig.2). From the resulting videos, the markers trajectories were followed with a video analyzer called Kinovea (Fig. 1).

A $300 \mathrm{~mm} * 300 \mathrm{~mm}$ force plate with a measuring frequency of $10000 \mathrm{~Hz}$ was buried under $100 \mathrm{~mm}$ of sand for the horse to land naturally on it. For the jumping experiment, the fence was placed at the right distance for the horse to land on it. The kinematics and ground reaction forces data were synchronized by analyzing the time of the video when the foot hit the ground and match it with the time of the force recording when the signal grows. The speed of the horse was recorded during the measurements with a speedometer.

\subsection{Gait analysis and compensation}

These noninvasive instrumentations led to the need of method developments for processing the resulting trajectories. The first level of correction needed is a compensation of the differences of speed between the horse and the camera. To correct these differences, a reference point was chosen under the saddle. As it was located on the horse's trunk, the horizontal speed of this point was supposed to represent the horizontal speed of the horse, so it should be horizontally motionless on the camera image if the horse and the camera had the same speed. Its abscise coordinates were therefore subtracted to the abscise coordinates of all other markers (Eq. (1)).

In Eq. (1), $Z_{\text {corr_ref }}(t)$ is the new abscise of the marker after correction, $Z(t)$ is the initial abscise of the marker, and $Z_{r e f}(t)$ is the abscise of the point under the saddle.

After correcting the speed differences between the horse and the camera, the effect of the mean speed of the horse, $v$ was introduced in the horizontal coordinates using Eq. (2). This enabled to obtain trajectories with the horse moving horizontally instead of running on the spot. 


$$
Z_{\text {spatial }}(t)=Z_{\text {corr_ref }}(t)+\mathrm{v} \times t
$$

In Eq. (2), $Z_{\text {corr_ref }}(t)$ is the abscise coordinate calculated in the previous correction, $v$ is the speed of the horse and $t$ is the time running. $Z_{\text {spatial }}(t)$ is the resulting abscise from adding the speed of the horse to get horizontal movement of the horse in the space.

These resulting trajectories showed that the foot stance phase during the ground contact was not on a same horizontal line for all strides. This error was corrected by imposing $Z_{P_{7}}(t)=0$ at each stance phase. After these corrections, another measurement error was observed: the distances between markers were not constant across time. These errors were due to the varying distance between the horse and the camera on the one hand and to skin artifact on the other hand. The skin artifact represents the difference of trajectory between the marker on the skin of the horse and the real movement of the bone under the soft tissues. The correction used, here, was derived from the method proposed by Cheze et al. ${ }^{[17]}$ : a length constraint value was defined on each segment between the markers and this value had to repeat itself across time. The reference length constraint value was defined at the timet $t_{r e f}$ for which the distances between markers were the closer to the lengths measured directly on the horse. This method is called a solidification procedure and mathematically, the Eq. (3), Eq. (4) and Eq. (5) need to be respected at all time.

$$
\begin{aligned}
|| \boldsymbol{P}_{\boldsymbol{i}} \boldsymbol{P}_{\boldsymbol{i}+\mathbf{1}}(t) \| & =|| \boldsymbol{P}_{\boldsymbol{i}} \boldsymbol{P}_{\boldsymbol{i}+\mathbf{1}}\left(t_{r e f}\right)|| \\
|| \boldsymbol{P}_{\boldsymbol{i}} \boldsymbol{S}_{\boldsymbol{j}}(t)|| & =|| \boldsymbol{P}_{\boldsymbol{i}} \boldsymbol{S}_{\boldsymbol{j}}\left(t_{r e f}\right)|| \\
|| \boldsymbol{S}_{\boldsymbol{j}} \boldsymbol{P}_{\boldsymbol{i}+\mathbf{1}}(t) \| & =|| \boldsymbol{S}_{\boldsymbol{j}} \boldsymbol{P}_{\boldsymbol{i}+\mathbf{1}}\left(t_{r e f}\right)||
\end{aligned}
$$

In Eq. (3), Eq. (4) and Eq. (5), $P i$ represents the principal marker at the joint $i$ ( $i=1$ to7), and $S_{j}$ represents the secondary marker between $P_{i}$ and $P_{i+1}(j=1$ to 4$)$.This final correction was applied for each segment and enabled to correct both problems of varying distance between horse and camera and skin artifact. The complete procedure of correction used for our gait experiments is summarized in Fig. 3.

\subsection{Geometry of bones}

In literature, authors often used CT tomography ${ }^{[1]}$, or MRI on cadavers ${ }^{[2]}$ to obtain the bones geometry. In this study, an industrial optical 3D digitizer called GOM ATOS 3 was used to scan the geometry of the forelimb bones of a horse skeleton. The numerical data of bone geometry were uploaded on CATIA V5 software in order to fit to the dimensions of the horse used for kinematics experiments. The distances between the principal markers are supposed to correspond to the bones lengths. This allows to deduce a value of scale factor $(\mathrm{f}=1.2)$. On CATIA V5 software, the surfaces were filled and assigned with a homogenous material with the same mean density as bone $\left(\rho_{\text {bones }}=1800 \mathrm{~kg} \cdot \mathrm{m}^{-3}\right)$.

\subsection{Kinematic modelling}

Our forelimb skeleton model consisted of six segments: the scapula, the humerus, the radius and ulna combined, the carpal bones combined with the metacarpal bone and the sesamoid bones, the proximal phalanx (P1), and finally the distal phalanx and middle phalanx (P2-P3) combined. These choices of combinations led to a model with five joints: shoulder, elbow, wrist, metacarpophalangeal (MCP) joint, interphalangeal joint (P2-P1). These joints were modeled with a ball in socket joint at the shoulder and revolute joints at the other articulations ${ }^{[25]}$. As the kinematics is known in $2 \mathrm{D}$, the ball in socket would be simplified as a revolute joint leading to a five degree-of-freedom model. To determine the joints axes of rotation, the surfaces of each articulation were best fitted with simple surfaces with CATIA V5 software (Fig. 4). For the shoulder, the center of the sphere was therefore chosen as the center of the joint and the axis of rotation, $z_{\text {shoulder }}$ was chosen normal to the plane of movement. For the hinge joints like the elbow joint, contact surfaces were best fitted with cylinders, defining the cylinder center as the frame center and the cylinder axis as rotation axis. It is supposed in that work that the both 
specimens of horse studies have no specific abnormal characteristic that would generate significant difference in the axis orientation of the articulation.

\subsection{Musculoskeletal modelling}

Muscles, tendons and ligaments were manually designed in CATIA V5 software using geometry and insertion information from the 3D Horse Anatomy of Biosphera software. The resulting surfaces of the muscles were filled in order to assign them a material with muscles density $\left(\rho_{\text {muscles }}=1070 \mathrm{~kg} . \mathrm{m}^{-3}\right)$. The muscles were then scaled and attached on the skeleton enabling to deduce the coordinates of the insertion points and the inertial properties of each segment (Fig. 5).

NMS Builder was used to build an OpenSim Model ${ }^{[11]}$ actuated by the 23 muscle-tendons and 5 ligamentous passive elastic structures defined before. Large muscles were represented with several strides to improve their efficiency due to the variation of angle between fibers and moment arms. The force-length curves of each tendon and ligament were modeled by fitting with the experimental data found in the literature ${ }^{[19-21]}$. The fiber length and pennation angles of most muscles were based on the literature ${ }^{[22-24]}$. For the muscles that were not studied in previous papers, the properties were chosen in a coherent manner according to the size, the location and the role of the muscle. The physiological crosssectional area (PCSA) of each muscle was determined from its 3D modelling on CATIA V5 and the maximum isometric force of each muscle was recalculated from its value of PCSA using a maximum isometric stress of $35 \mathrm{~N} / \mathrm{cm}^{2}{ }^{[2-25]}$ (Eq. (6)).

$$
\mathrm{F}_{\text {iso }_{\text {max }}}=\mathrm{PCSA} * \sigma_{\text {iso }_{\text {max }}}(6)
$$

The tendons slack lengths were measured from the CAD-modeling of muscles. Wrapping surfaces (spheres or cylinders) were located at strategic locations to constraint the soft structures to get around bones and not to pass through them. The final musculoskeletal is represented in Fig. 5.

\subsection{Dynamic computations}

The angular variations at joints during locomotion were computed using the inverse kinematics module of OpenSim software. This tool enables to best fit the angular variations of joints with the coordinates of each markers. Then, the inverse dynamics tool of OpenSim software was used to deduce the moments at joints taking into account the ground reaction force, the mechanical inertias and the masses of the musculoskeletal model. The forces in the muscles of the model and their activations were computed using the Static Optimization tool of OpenSim and the joint reactions were then computed.

\subsection{Adjustments of the musculoskeletal model according first dynamic computations}

Generally, the forces generated by muscles parameters are not high enough to reach the given kinematics and moments reserves would be added by OpenSim to satisfy the fundamental equations of the Dynamics. These reserves would affect joint reactions loads calculations and should be as small as possible. In this work, some strategies were used to reduce reserves values. First, large muscles were modelled with at least three strides. The joint presenting the highest reserves was then identified and blocked to better manage the complexity of kinematics. Then, the active muscles at each articulation were identified and compared to the activations recomputed by OpenSim. The activation is directly linked to the tendon slack length: if the length value is too small or too big, the muscle might not activate at all. The tendon lengths were optimized on these muscles and their impact on the joint reserve was investigated. Then, the maximal isometric forces of muscles were increased for the muscles affecting the joint until the reserves were maximally reduced. Fig. 6 summarizes the different methods for adjustment of the musculoskeletal model.

\section{Results}

\subsection{Kinematics results}


The horse trotted at a mean speed of $4 \pm 0.1 \mathrm{~m} . \mathrm{s}^{-1}$ and at jumping, the speed of the body was about $5 \mathrm{~m} . \mathrm{s}^{\mathrm{s}}$ ${ }^{1}$. The trajectories were corrected according to Fig.3and the solidification procedure resulting trajectories are shown in Fig.7. The inverse kinematics tool of OpenSim enabled to determine the angular variations at each joint, which are represented in Fig. 8. At rest, the shoulder angle is opened of $125 \mathrm{deg}$, and at trotting it varies from $-3.8 \%$ to $+15.6 \%$ around this value. The elbow is initially opened of $150 \mathrm{deg}$, and it closes up to $-37.7 \%$ and opens up to $+6.5 \%$. The initial position of the wrist is $175 \mathrm{deg}$ and across trotting it goes from $-39.1 \%$ to $+8.6 \%$ of this value. At rest, the MCP joint is opened with $154 \mathrm{deg}$, and at trotting it closes only to $-1 \%$ but opens up to $+54.9 \%$. Finally, the P2-P1 angle has an initial opening of $172 \mathrm{deg}$, and closes up to $-0.8 \%$ but opens up to $+28.2 \%$.

At jumping, the angular variations are globally wider. At the shoulder joint, the angle varies from $-13 \%$ to $+7.6 \%$, at elbow joint it varies from $-53 \%$ to $+6.4 \%$ and at wrist joint it goes from $-57.6 \%$ to $+9.3 \%$. The MCP joint closes up to $-12.7 \%$ and opens up to $+48.3 \%$ and finally, the P2-P1 angle varies around its rest position from $0.87 \%$ to $28.8 \%$.

\subsection{Ground reaction forces results}

The measured ground reaction forces in the three directions at trotting and jumping are given in Fig. 9. The maximal vertical forces at trotting and jumping on the sand are respectively: $9.19 \mathrm{~N} / \mathrm{kg}$ and 11.69 $\mathrm{N} / \mathrm{kg}$. It can be observed that the contact force at jumping is about $27 \%$ higher and also that the contact time is shorter.

\subsection{Results of dynamic simulations: reserves reductions}

With the initial degree-of-freedom and muscles configurations, joints showed high reserves at trotting, especially for shoulder and MCP joints (Fig. 10.a). The MCP rotation was blocked at its initial position (154 deg) because it presented the higher reserve. Before validating the reserves reduction with the rotation blocking method, the kinematics were analyzed to verify that the blockage of this rotation did not affect the kinematics of other joints. Very few differences were observed, the worst being at the MCP marker with $0.037 \mathrm{~m}$, for a horse of $1.70 \mathrm{~m}$ high, representing therefore only $2.2 \%$ of its height. After modifying the kinematics, the muscles that should be active at each joint were identified and their parameters were changed in order to reduce the reserves. The resulted reserves values for trot are given in Fig. 10.b and Table 1 and the muscular parameters changes are summarized in Table 2. The reserves were reduced to almost zero, meaning that the final model dynamic resolution can handle the imposed ground reaction forces and movement without any artificial torque addition.

Concerning the reserves at jumping, they were much higher and the trotting muscular parameters were not sufficiently high to reduce the reserves at jumping (Fig. 10.d). The parameters were therefore modified (Table 3 ) and the values of reserves in the jumping study were reduced at least from $66 \%$ (Fig 10.e and Table 1). When running the trotting simulation with jumping muscular parameters, the resulting reserves were still very low (Fig. 10.c), which validated the use of these muscular parameters for trotting simulation too. Fig. 11 compares the computation of joint reactions forces at trotting with trotting and jumping muscular parameters. These values are very similar, especially for shoulder, MCP and P2-P1 joints.

\subsection{Results of dynamic simulations: joint loadings}

The norms of the joint reaction forces computed by OpenSim for the trot simulation are given in Table 4 and Fig.12.a. The highest maximal contact force is at the wrist joint with $50.4 \mathrm{~N} / \mathrm{kg}$, followed by the elbow joint at $39.2 \mathrm{~N} / \mathrm{kg}$ and the MCP joint at $34.9 \mathrm{~N} / \mathrm{kg}$. The joint reaction force at the shoulder is the lowest with $21.7 \mathrm{~N} / \mathrm{kg}$, close to the P2-P1 joint reaching $28.2 \mathrm{~N} / \mathrm{kg}$. At jumping (Fig. 10.b), the values of joint reaction forces were higher with $47.5 \mathrm{~N} / \mathrm{kg}$ at the shoulder, $74.2 \mathrm{~N} / \mathrm{kg}$ at the elbow, $78.5 \mathrm{~N} / \mathrm{kg}$ at the wrist, $58.9 \mathrm{~N} / \mathrm{kg}$ at the MCP and $58.6 \mathrm{~N} / \mathrm{kg}$ at the P2-P1 joint. The results of Table 4 indicate that the vertical joint loading increased a lot from trotting to jumping with at least $45.2 \%$ of increase for the P2-P1 joint and more for the others, whereas the ground reaction force increased of only $27 \%$ from 9.19 $\mathrm{N} / \mathrm{kg}$ to $11.69 \mathrm{~N} / \mathrm{kg}$. The horizontal component of the joint reactions did not show a similar evolution across joints between trotting and jumping. These differences of increase percentages for the vertical component and of evolution for the horizontal component are linked to the high differences of 
kinematics, speeds, and angular evolutions of joints between trotting and jumping. The norms and directions of the computed forces are represented in (Fig. 13) for both trotting and jumping.

\section{Discussion}

The computed results for trotting are compared to literature in order to check our model. Our measurements of joint angles are very close to previous studies ${ }^{[26]}$ and this enables to validate the correction methods applied to the kinematics data. Peak shoulder and elbow joints angles measured for trotting were $144.5 \mathrm{deg}$ and $159.7 \mathrm{deg}$ respectively, which are very similar to the values reported by Dutto et al. ${ }^{[26]}(138 \pm 5 \mathrm{deg}$ and $150 \pm 10 \mathrm{deg})$. For the wrist and MCP joints, the measured values are $190.9 \mathrm{deg}$ and $238.5 \mathrm{deg}$ respectively and those are also very close to the values given by Dutto et al. ${ }^{[26]}$ (186 $\pm 9 \mathrm{deg}$ and $231 \pm 4 \mathrm{deg}$ ) and also by Harrison et al. ${ }^{[2]}$ (182 $\pm 2 \mathrm{deg}$ and $241 \pm 4 \mathrm{deg}$ ). Finally, for the phalangeal joint, the measured value was $220.5 \mathrm{deg}$, which shows high similarity with Dutto et al. [26] who reported a value of $220 \pm 3 \mathrm{deg}$. At jumping, the joint angular variations intervals were larger than at trotting, due to wider movements to reach the jumping kinematics. These intervals are approximatively the same for shoulder and P2-P1 joints. For the elbow, the wrist and the MCP joints, the articulations close and open more at jumping than at trotting.

The joint loading values at trot are quite different from the values found by Harrison et al. ${ }^{[2]}$ for the comparable values at MCP (40.6 N/kg for Harrison et al. ${ }^{[2]}, 34.9 \mathrm{~N} / \mathrm{kg}$ for us) and wrist (28 N/kg for Harrison et al. ${ }^{[2]}, 50.4 \mathrm{~N} / \mathrm{kg}$ for us) joints (Fig 12.a). This can be explained by a higher speed (4 m.s ${ }^{-1}$ for us against $1.4 \mathrm{~m} \cdot \mathrm{s}^{-1}$ for Harrison), because the increase of speed causes higher ground reaction force to keep the position against gravity ${ }^{[27]}$ and this leads to higher moments at MCP and wrist ${ }^{[26]}$ causing higher joint loadings. This can also be explained by the difference of ground material: sand in our study instead of rubber matting or turf track for Harrison et al. ${ }^{[2]}$. The modeling simplifications and the reduction of reserves also play a major role on the joint reactions.

Another interesting aspect is the significant link between angular opening and joint loading. For the elbow and the wrist joints which presented the largest angular openings with 84.3 deg for elbow and $86.1 \mathrm{deg}$ for wrist at trot, it was observed that they also presented the highest joint loadings of the limb with $39.2 \mathrm{~N} / \mathrm{kg}$ for elbow and $50.4 \mathrm{~N} / \mathrm{kg}$ for wrist at trot. This was also observable at jumping.

This study presented some limitations in terms of measurements and modelling methods. Our kinematics measurement methods were fast and simple to settle and non-invasive at all but it needed important corrections after all. To validate the measurement method, it could be interesting to compare the resulting trajectories with trajectories from Vicon or other techniques measurements. The kinematics errors lead to large nonphysical forces. In OpenSim a Residual Reduction Algorithm function enables to minimize the effects of modeling and marker data processing errors using a numerical optimization independent from error sources brought to the fore in the experiments. In this paper, the compensation methods were based on observed physical sources like differences of speeds or skin artifact. The application of the proposed compensation methods to the kinematic measurements reduced the best fit residues of OpenSim kinematics module without markers weighting, from 0.0168 to 0.0088 representing $48 \%$ of reduction. To test the effectiveness of this method, it has been compared to the results given by OpenSim's inverse kinematics module where the markers were weighted according to their relevance. This module computes a best fit residue of 0.0093 . The proposed method was considered efficient and retained for kinematic compensation for this specific kinematic measurement.

\section{Conclusion}

The overall goal of this study was to describe a method to determine the joint contact loading in the horse forelimb at high jerk dynamics like jumping. To our knowledge, this study is the first to estimate joint reaction forces in the forelimb of a horse at jumping. Non-invasive methods were used for the measurements of kinematics and ground reaction forces and this enabled to keep the horse in its usual environment and to optimize the repeatability of the results. The kinematics needed compensations because of the simplicity of our measurements. A musculoskeletal model was built with mechanical methods: the bones were scanned with the GOM ATOS 3 to retrieve their geometry on CATIA V5, a 
CAD software. The determination of the centers and directions of joints with CATIA V5 ensures reliability and accuracy. The 3D modelling of muscles enabled to avoid dissecting a horse cadaver. This modelling enabled to deduce the accurate positions of the insertion points and the inertial parameters of segments. This methodology can be easily reused for other experimental campaigns on other horse specimens. The dynamics calculations were first run at trotting to validate the model and then at jumping. As part of bio-inspiration, these results will contribute to design the bio-inspired joints.

\section{Acknowledgement}

We thank Arroyave Tobon Santiago, Thouveny Thomas and Perrotey Arthur for their precious help on this work. We also thank Avisse Tristan and Demassieux Jacques for the help on the experiments. We thank the GIR Laboratory of Airbus Helicopters members Erik Faravel and Hamiache Romain for their help on ground reaction forces measurements. Airbus Helicopters/Aix-Marseille Université Scientific Chair on Bio-Inspired Mechanical Design funded this work.

* The reference data can be found online at:

https://simtk.org/docman/?group_id=1728 


\section{References}

[1] Lerner Z F, Gadomski B C, Ipson A K, Haussler K K, Puttlitz C M, Browning R C. Modulating tibiofemoral contact force in the sheep hind limb via treadmill walking: predictions from an opensim musculoskeletal model. Journal of Orthopaedic Research, 2015, 33(8), 1128-1133.

[2] Harrison S M, Whitton R C, Kawcak C E, Stover S M, Pandy M G. Relationship between muscle forces, joint loading and utilization of elastic strain energy in equine locomotion. Journal of Experimental Biology, 2010, 213(23), 3998-4009

[3] Picault E, Mermoz E, Thouveny T, Linares J M. Smart pressure distribution estimation in biological joints for mechanical bio-inspired design. CIRP Annals, 2018.

[4] Paluska S A. An overview of hip injuries in running. Sports Medicine, 2005, 35(11), 991-1014.

[5] Boyd K T, Peirce N S, Batt M E. Common hip injuries in sport. Sports Medicine, 1997, 24(4), 273 288.

[6] D'Lima D D, Patil S, Steklov N, Slamin J E, Colwell Jr C W. Tibial forces measured in vivo after total knee arthroplasty. The Journal of arthroplasty, 2006, 21(2), 255-262.

[7] Walter J P, D'lima D D, Colwell Jr C W, Fregly B J. Decreased knee adduction moment does not guarantee decreased medial contact force during gait. Journal of Orthopaedic Research, 2010, 28(10), 1348-1354

[8] Li G, Gil J, Kanamori A, Woo S Y. A validated three-dimensional computational model of a human knee joint. Journal of biomechanical engineering, 1999, 121(6), 657-662.

[9] Donahue T L H, Hull M L, Rashid M M, Jacobs C R. A finite element model of the human knee joint for the study of tibio-femoral contact. Journal of biomechanical engineering, 2002, 124(3), 273-280.

[10] Pena E, Calvo B, Martinez M A, Doblare M. A three-dimensional finite element analysis of the combined behavior of ligaments and menisci in the healthy human knee joint. Journal of biomechanics, 2006, 39(9), 1686-1701.

[11] Delp S L, Anderson F C, Arnold A S, Loan P, Habib A, John C T, Thelen D G. OpenSim: opensource software to create and analyze dynamic simulations of movement. IEEE transactions on biomedical engineering, 2007, 54(11), 1940-1950.

[12] Arnold E M, Ward S R, Lieber R L, Delp S L. A model of the lower limb for analysis of human movement. Annals of biomedical engineering, 2009, 38(2), 269-279.

[13] Hamner S R, Seth A, Delp S L. Muscle contributions to propulsion and support during running. Journal of biomechanics, 2010, 43(14), 2709-2716.

[14] Reinbolt J A, Seth A, Delp S L. Simulation of human movement: applications using OpenSim. Procedia Iutam, 2011, 2, 186-198.

[15] Panagiotopoulou O, Rankin J W, Gatesy S M, Hutchinson J R. A preliminary case study of the effect of shoe-wearing on the biomechanics of a horse's foot. PeerJ, 2016, 4, e2164.

[16] Hutchinson, J. R., Rankin, J. W., Rubenson, J., Rosenbluth, K. H., Siston, R. A., \& Delp, S. L. (2015). Musculoskeletal modelling of an ostrich (Struthio camelus) pelvic limb: influence of limb orientation on muscular capacity during locomotion. PeerJ, 3, e1001.

[17] Cheze L, Fregly B J, Dimnet J. A solidification procedure to facilitate kinematic analyses based on video system data. Journal of biomechanics, 1995, 28(7), 879-884.

[18] Arnold E M, Ward S R, Lieber R L, Delp S L. A model of the lower limb for analysis of human movement. Annals of biomedical engineering, 2010, 38(2), 269-279.

[19] Swanstrom M D, Stover S M, Hubbard M, Hawkins D A. Determination of passive mechanical properties of the superficial and deep digital flexor muscle-ligament-tendon complexes in the forelimbs of horses. American journal of veterinary research, 2004, 65(2), 188-197.

[20] Swanstrom M D, Zarucco L, Hubbard M, Stover S M, Hawkins D A. Musculoskeletal modeling and dynamic simulation of the thoroughbred equine forelimb during stance phase of the gallop. Journal of biomechanical engineering, 2005, 127(2), 318-328.

[21] Swanstrom M D, Zarucco L, Stover S M, Hubbard M, Hawkins D A, Driessen B, Steffey E P. Passive and active mechanical properties of the superficial and deep digital flexor muscles in the forelimbs of anesthetized Thoroughbred horses. Journal of biomechanics, 2005, 38(3), 579-586.

[22] Brown N A, Pandy M G, Kawcak C E, McIlwraith C W. Force-and moment-generating capacities of muscles in the distal forelimb of the horse. Journal of Anatomy, 2003, 203(1), 101-113. 
[23] Watson J C, Wilson A M. Muscle architecture of biceps brachii, triceps brachii and supraspinatus in the horse. Journal of anatomy, 2007, 210(1), 32-40.

[24] Payne R C, Veenman P, Wilson A M. The role of the extrinsic thoracic limb muscles in equine locomotion. Journal of Anatomy, 2004, 205(6), 479-490.

[25] Zajac F E. Muscle and tendon Properties models scaling and application to biomechanics and motor. Critical reviews in biomedical engineering, 1989, 17(4), 359-411.

[26] Dutto D J, Hoyt D F, Clayton H M, Cogger E A, Wickler S J. Joint work and power for both the forelimb and hindlimb during trotting in the horse. Journal of Experimental Biology, 2006, 209(20), 3990-3999.

[27] Dutto D J, Hoyt D F, Cogger E A, Wickler S J. Ground reaction forces in horses trotting up an incline and on the level over a range of speeds. Journal of Experimental Biology, 2004, 207(20), 3507-3514. 


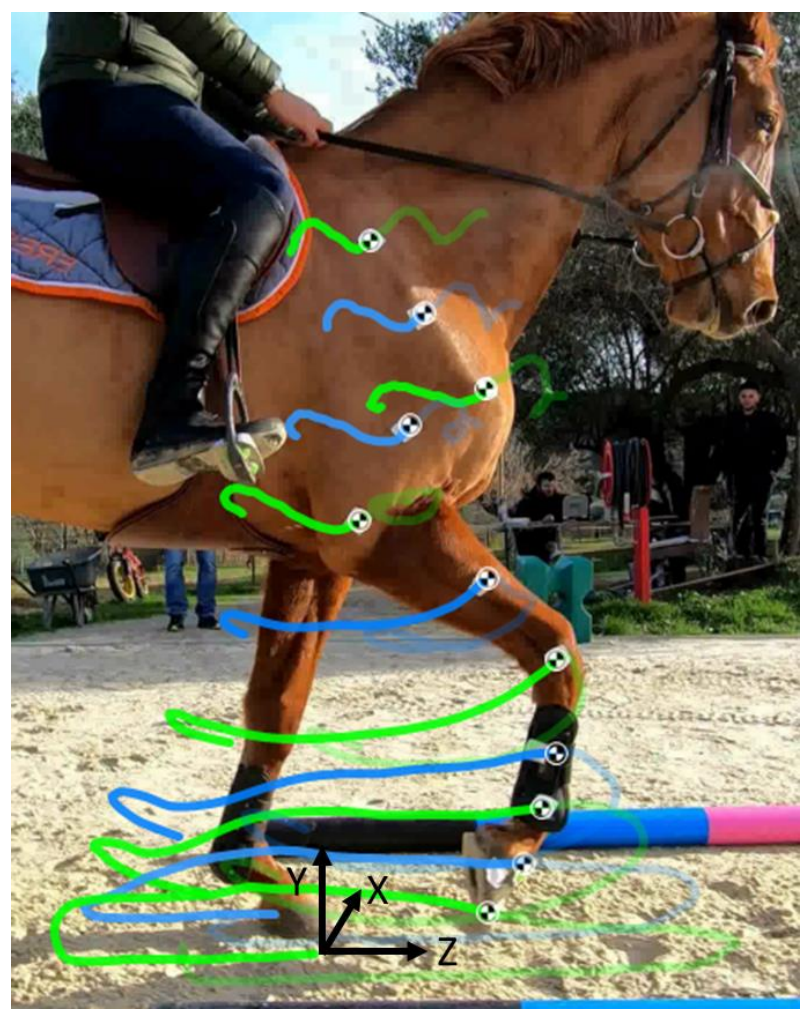

Fig. 1 Right forelimb equipped with the markers followed by Kinovea software. The green markers are the principal markers and the blue ones are the secondary markers. The secondary markers were placed between the principal markers but unaligned with them. 


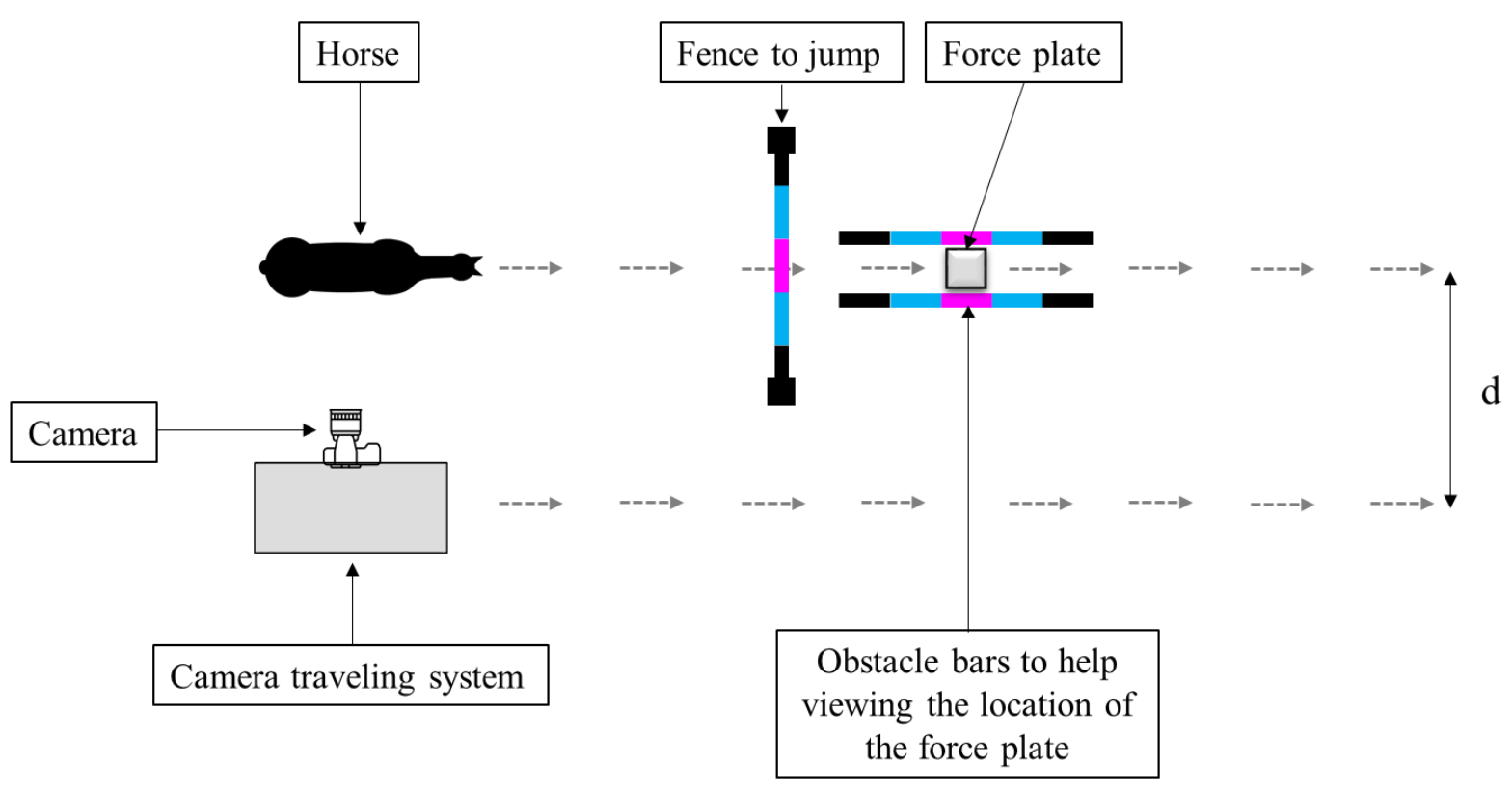

Fig. 2 Top view of the running experiment. The horse moved along a virtual line, followed by the camera at a distance d. It passes over a force plate buried in the sand. At jump, a fence was placed before the force plate. The distance was readjusted after several passages. 


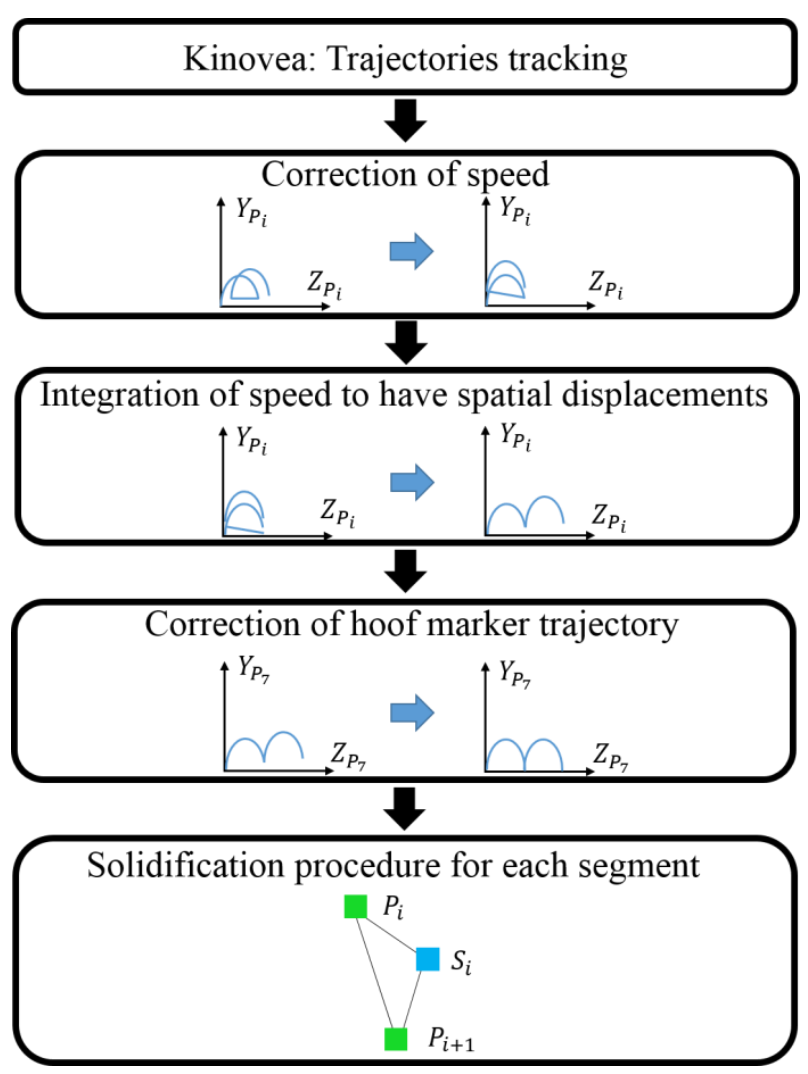

Fig. 3 Procedure of correction of the trajectories. $P_{i}$ represents the principal marker at the joint $i$, and $S_{i}$ represents the secondary marker between $P_{i}$ and $P_{i+1}$

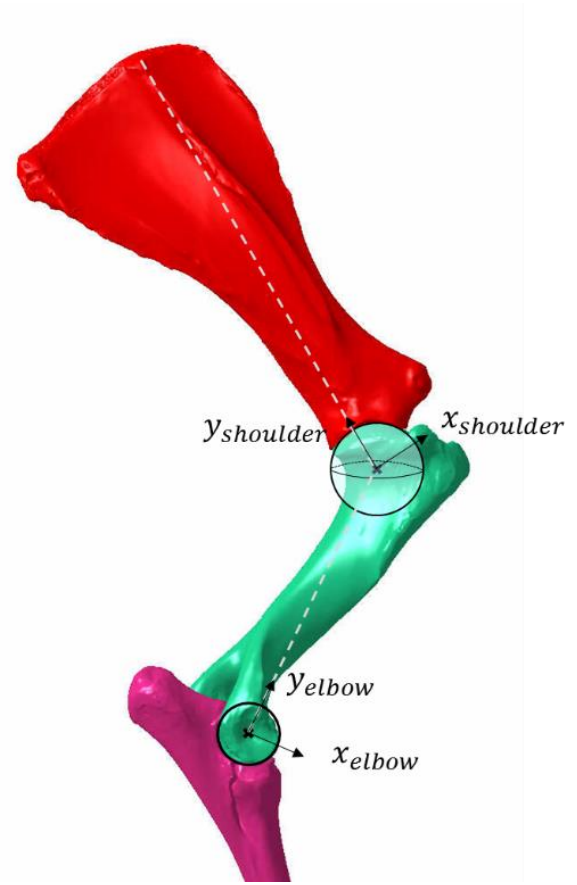

Fig. 4 Methodology to find the joint local frames. Examples of the shoulder and the elbow. 


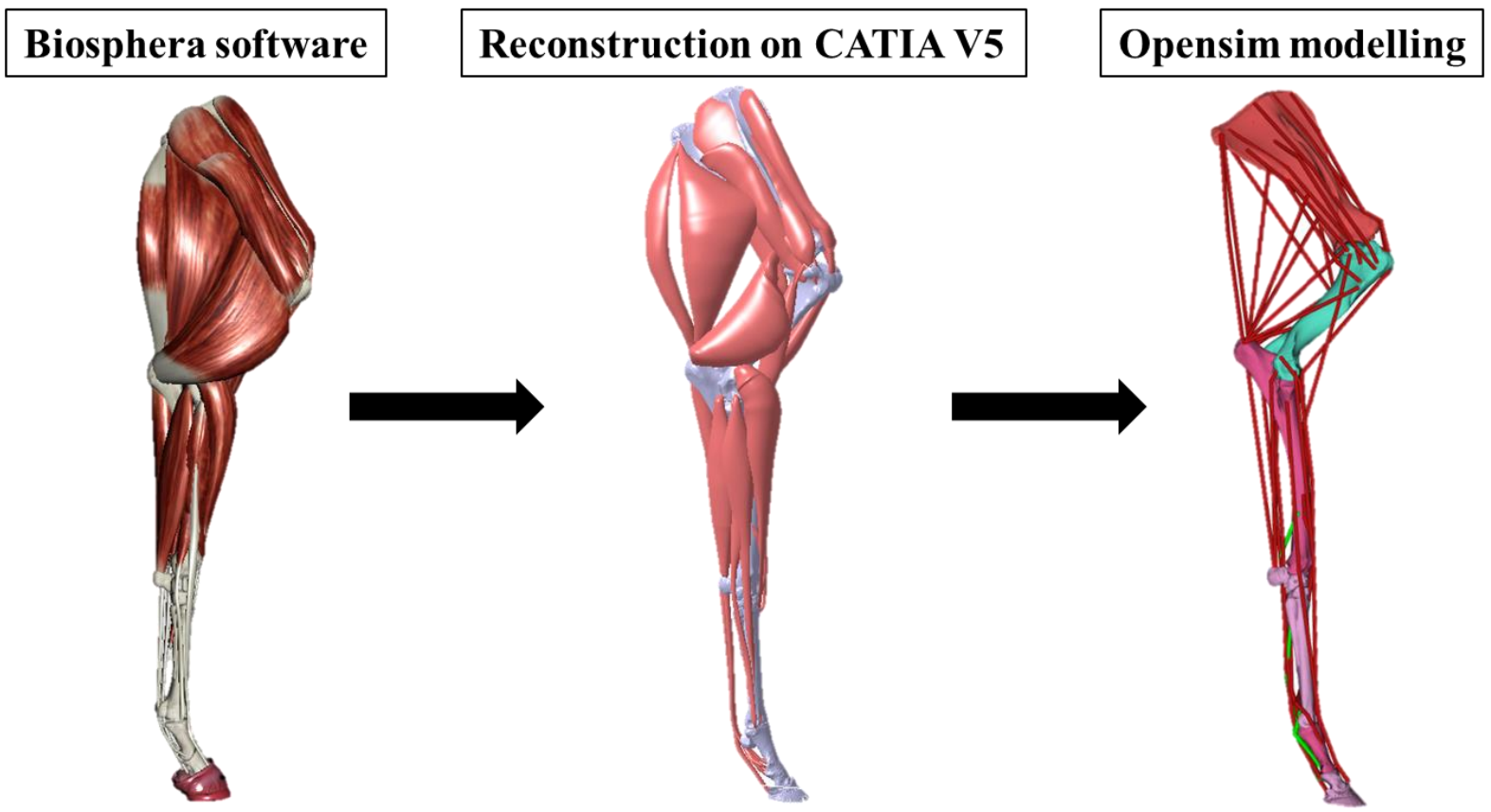

Fig. 5 Reconstruction of the entire forelimb on CATIA V5 and construction of the OpenSim model from the Biosphera Software (without the wrapping surfaces). 


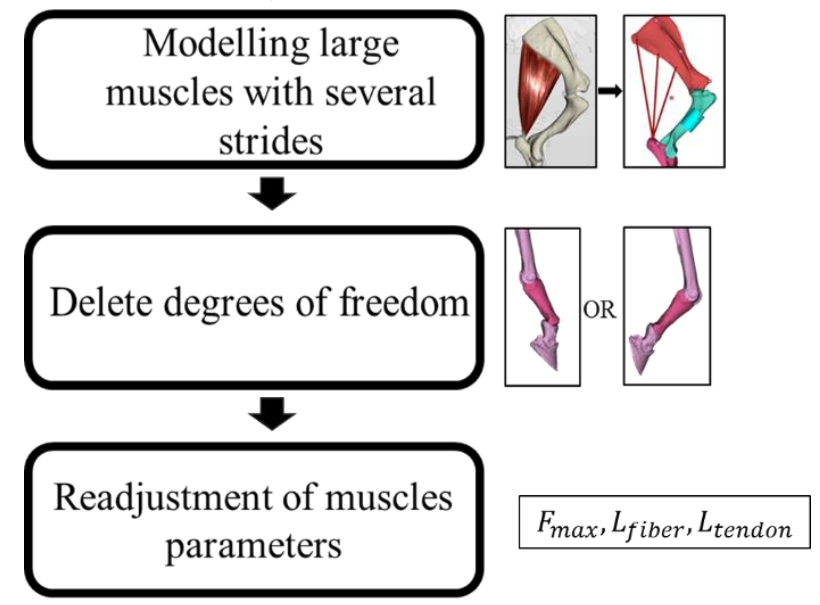

Fig. 6 Solutions for adjusting the model. 


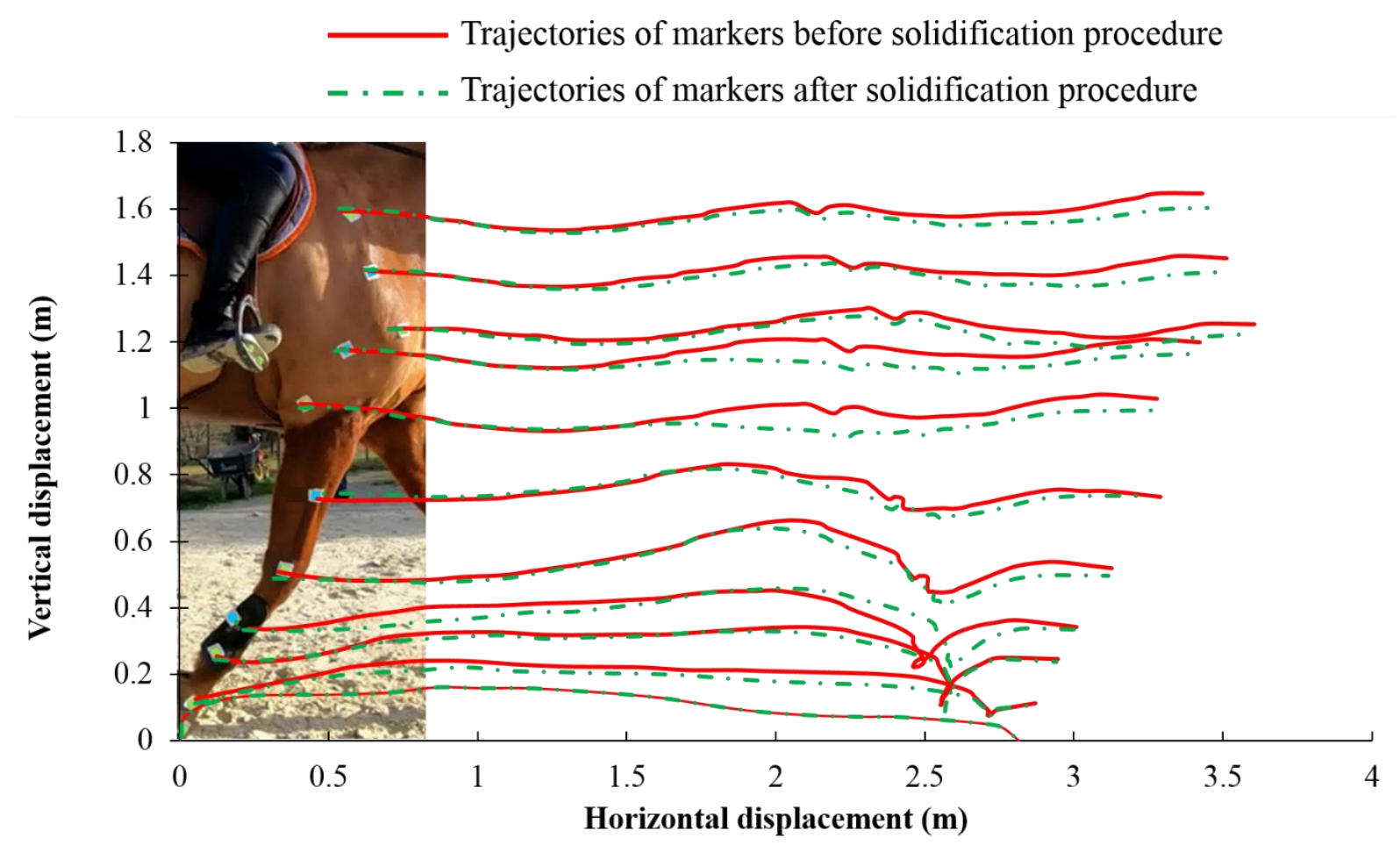

Fig. 7 Trajectories of the markers before and after applying the solidification procedure. 


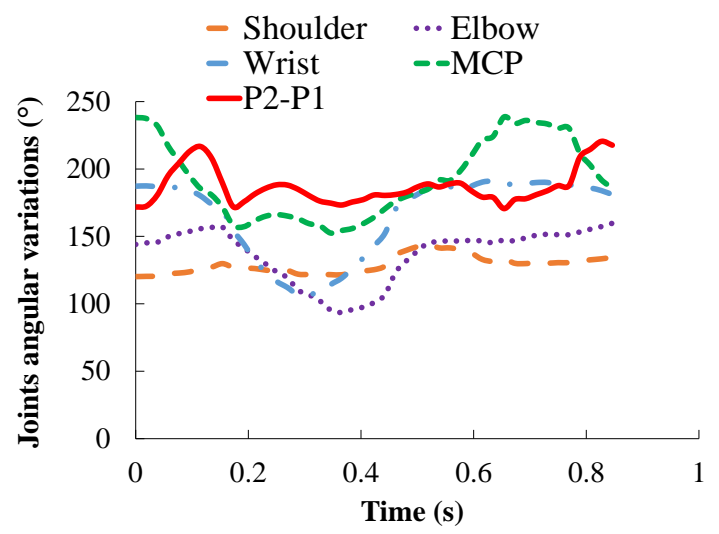

(a)

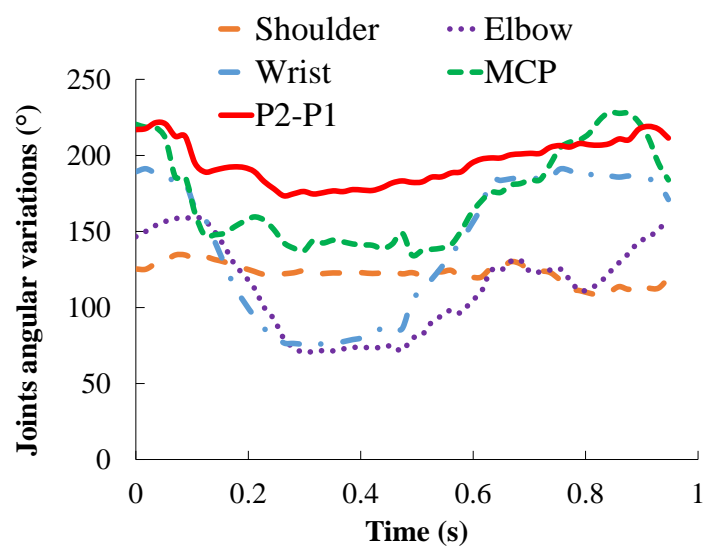

(b)

Fig. 8 Joints angular variations across time. (a) At trotting. (b) At jumping. 


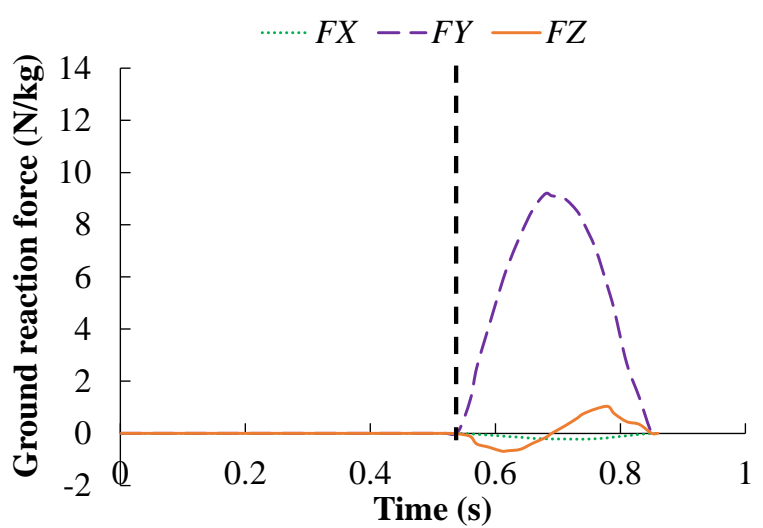

(a)

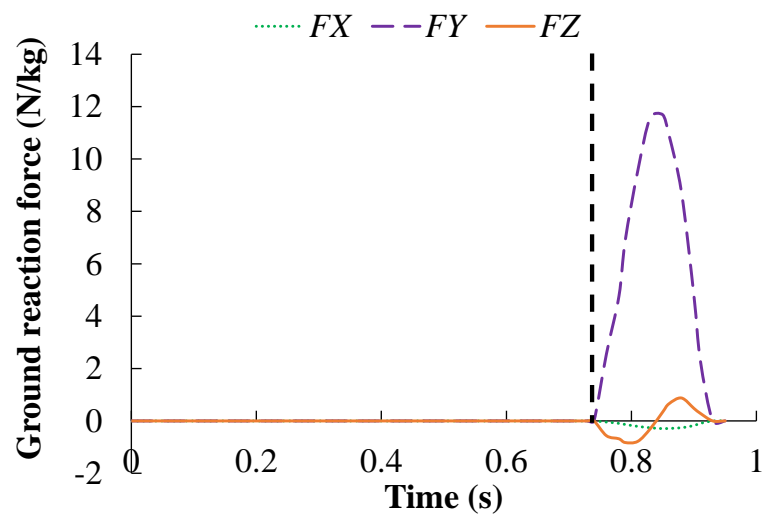

(b)

Fig. 9 Ground reaction forces measured at trot (a) and at jumping a fence of $1 \mathrm{~m}$ high (b). The black dashed line represents the limit between swing and stance phases. 

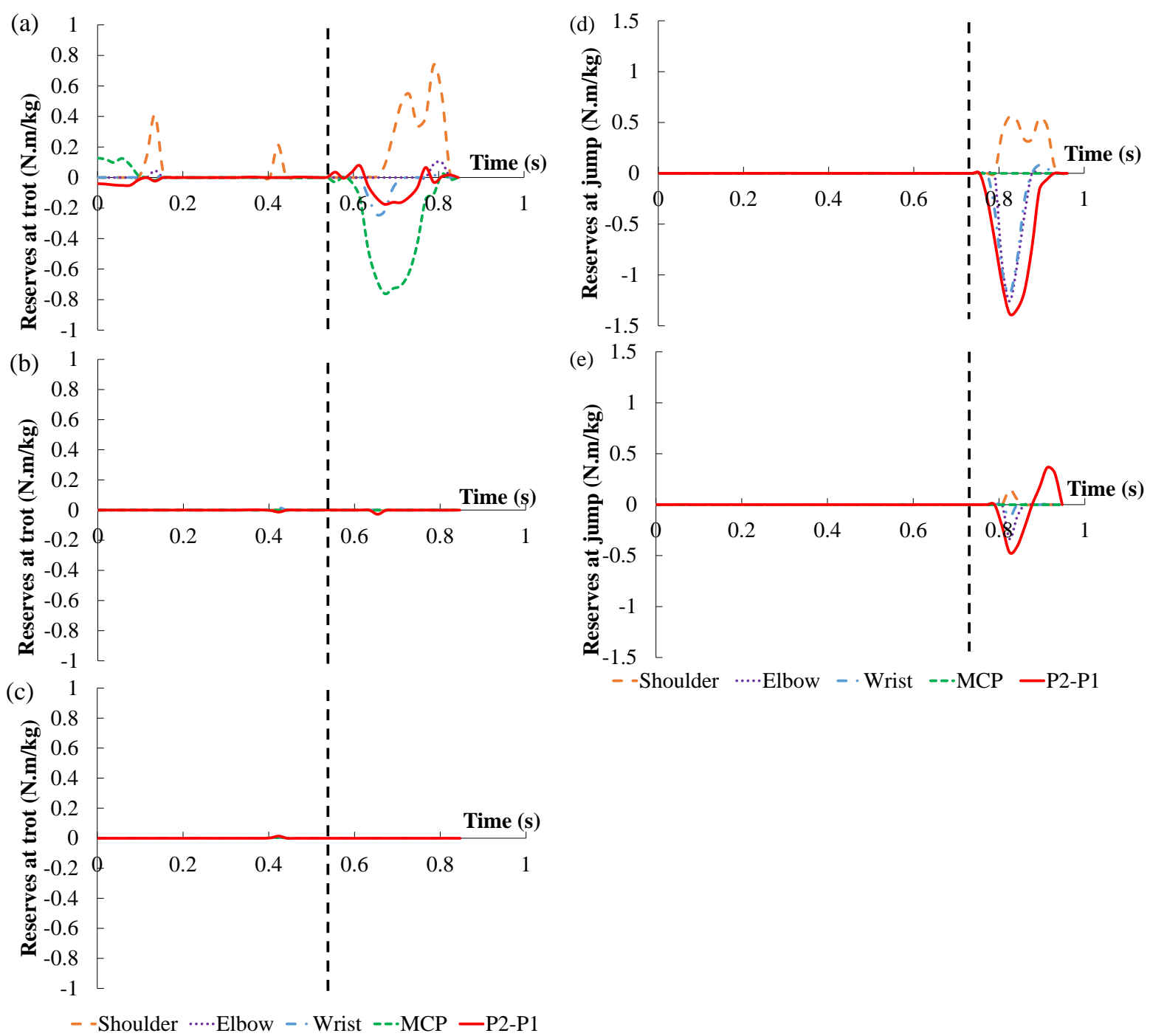

Fig. 10 Reserves at trot: (a) Before adjustments. (b) After adjustments. (c) After applying the jumping model adjustments. Reserves at jump. (d) Before adjustments with the final configuration of trot. (e)

After adjustments. The black dashed line represents the limit between swing and stance phases. 


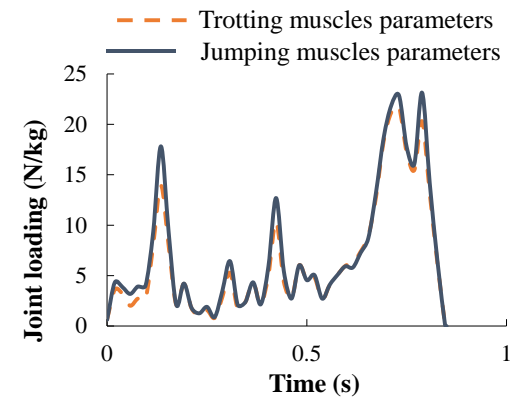

(a)

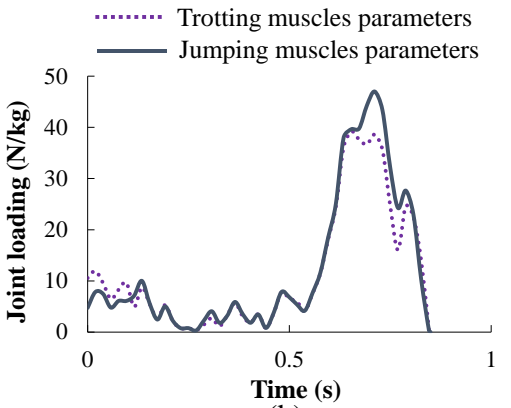

(b)

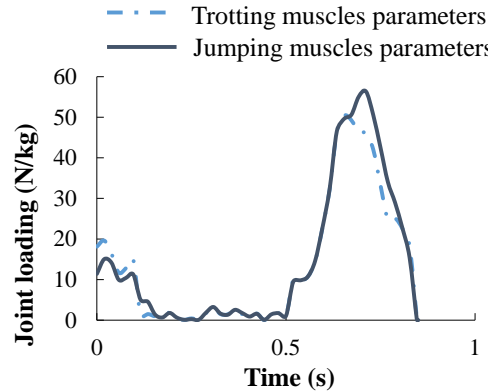

(c)

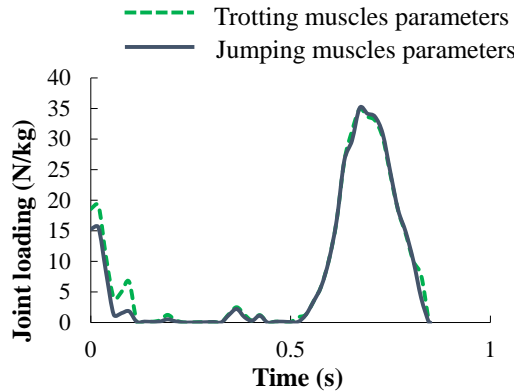

(d)

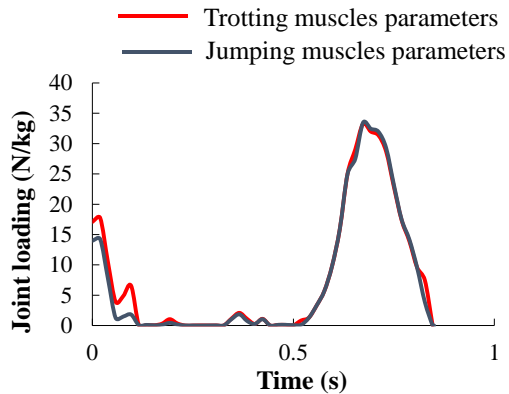

(e)

Fig. 11 Comparison of the vertical joint loading results for the trotting parameters (Table 2) and the jumping parameters (Table 3). (a) Shoulder vertical reaction force. (b) Elbow vertical reaction force. (c) Wrist vertical reaction force. (d) MCP vertical reaction force. (e) P2-P1 vertical reaction force.

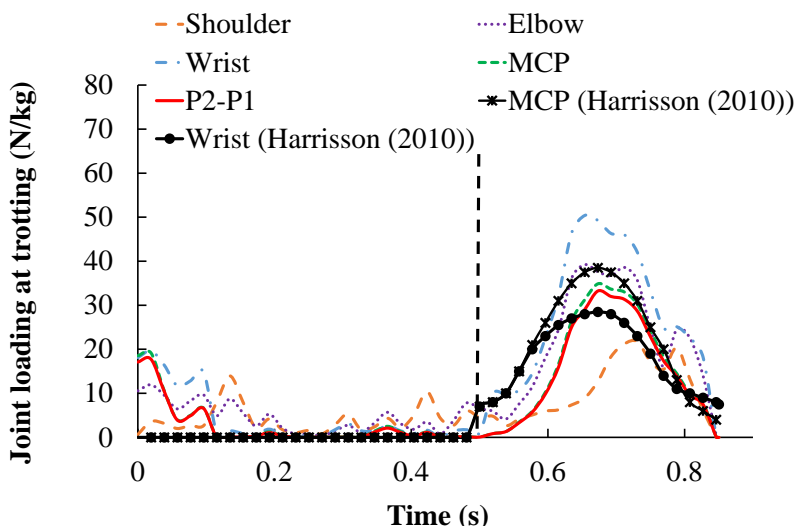

(a)

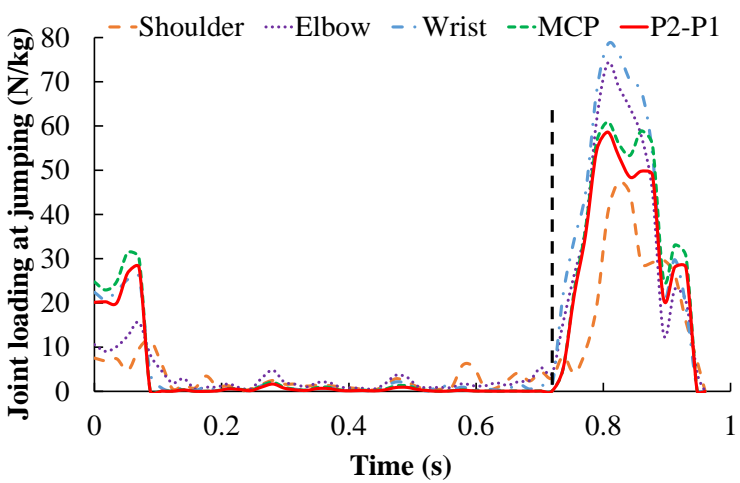

(b)

Fig. 12 Results of joint loading for trotting (a) and jumping (b). For trotting, the results are compared to Harrison (2010) values. The black dashed line represents the passage from swing to stance phase. 


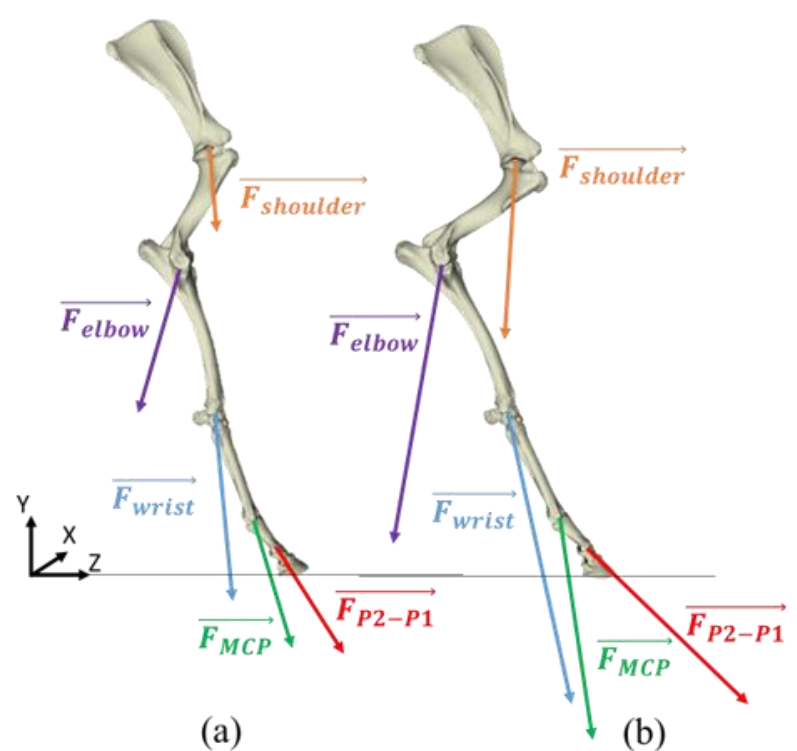

Fig. 13 Representation of the maximal joint reaction forces in the right forelimb of a horse (a) at trotting and (b) at jumping a $1 \mathrm{~m}$ fence. 


\begin{tabular}{|c|c|c|c|c|c|c|c|c|c|}
\hline & \multicolumn{3}{|c|}{ Trot reserves absolute values $(\mathrm{N} . \mathrm{m} / \mathrm{kg})$} & \multicolumn{3}{|c|}{ Jump reserves absolute values (N.m/kg) } & \multicolumn{3}{|c|}{$\begin{array}{c}\text { Trot reserves absolute values }(\mathbf{N} . \mathbf{m} / \mathbf{k g}) \\
\text { with jumping parameters } \\
\end{array}$} \\
\hline & Initial model & Final model & Decrease $(\%)$ & Initial model & Final model & Decrease $(\%)$ & Initial model & Final model & Decrease $(\%)$ \\
\hline Shoulder & 0.73 & $5.40 \mathrm{E}-04$ & -99.9 & 0.56 & 0.15 & -73.2 & 0.73 & $8.36 \mathrm{E}-05$ & -100.0 \\
\hline Elbow & 0.1 & $7.60 \mathrm{E}-05$ & -99.9 & 1.26 & 0.34 & -73.0 & 0.1 & $8.76 \mathrm{E}-05$ & -99.9 \\
\hline Wrist & 0.24 & 0.016 & -93.3 & 1.19 & 0.12 & -89.9 & 0.24 & 0.01501 & -93.7 \\
\hline $\mathrm{MCP}$ & 0.76 & 0 & -100.0 & 0 & 0 & 0.0 & 0.76 & 0 & -100.0 \\
\hline P2-P1 & 0.18 & 0.028 & -84.4 & 1.38 & 0.47 & -65.9 & 0.18 & 0.014 & -92.2 \\
\hline
\end{tabular}

Table 1. Maximal reserves values at trot and jumping for the initial and final models. The decrease of reserves between the initial and the final models are given in $\%$.

\begin{tabular}{|c|c|c|c|}
\hline \multicolumn{3}{|c|}{ Changes from initial model } & \\
\hline Muscle & $\begin{array}{l}\text { Maximal isometric force } \\
(\mathbf{N})\end{array}$ & $\begin{array}{c}\text { Tendon length } \\
(\mathrm{mm})\end{array}$ & $\begin{array}{c}\text { Joint } \\
\text { concerned }\end{array}$ \\
\hline Biceps & & 750 & $\begin{array}{l}\text { Shoulder, } \\
\text { Elbow }\end{array}$ \\
\hline Brachialis & & 200 & Elbow \\
\hline Deep digital flexor & 5000 & & $\begin{array}{l}\text { Elbow, Wrist, } \\
\text { MCP, P2-P1 }\end{array}$ \\
\hline Extensor carpii ulnaris & & 250 & Wrist \\
\hline Subscapularis & 2000 & & Shoulder \\
\hline Superficial digital extensor & 6000 & 750 & $\begin{array}{l}\text { Elbow, Wrist, } \\
\text { MCP, P2-P1 }\end{array}$ \\
\hline Supraspinatus & & 100 & Shoulder \\
\hline
\end{tabular}

Table 2. Changes applied to the muscles to reduce the reserves at trot. 


\begin{tabular}{|c|c|c|c|}
\hline \multicolumn{3}{|c|}{ Changes from trot model (Table 2) } & \multirow[b]{2}{*}{$\begin{array}{c}\text { Joint } \\
\text { concerned }\end{array}$} \\
\hline Muscle & $\begin{array}{l}\text { Maximal isometric force } \\
(\mathrm{N})\end{array}$ & $\begin{array}{c}\text { Tendon length } \\
(\mathrm{mm})\end{array}$ & \\
\hline Common digital extensor & 5000 & 700 & $\begin{array}{c}\text { Wrist, MCP, } \\
\text { P2-P1 }\end{array}$ \\
\hline Deep digital flexor & 10000 & & $\begin{array}{l}\text { Elbow, Wrist, } \\
\text { MCP, P2-P1 }\end{array}$ \\
\hline Flexor carpii ulnaris & 7000 & 150 & Wrist \\
\hline Subscapularis & 5000 & & Shoulder \\
\hline $\begin{array}{c}\text { Superficial digital } \\
\text { extensor }\end{array}$ & 10000 & & $\begin{array}{l}\text { Elbow, Wrist, } \\
\text { MCP, P2-P1 }\end{array}$ \\
\hline Supraspinatus & 5000 & & Shoulder \\
\hline Triceps medial head & 2000 & & Elbow \\
\hline
\end{tabular}

Table 3. Changes applied to the muscles to reduce the reserves at jumping.

\begin{tabular}{|c|c|c|c|c|c|c|c|c|c|}
\hline & \multicolumn{4}{|c|}{ Joint loading } \\
\hline \multirow{2}{*}{ Joint } & \multicolumn{3}{|c|}{ Trot (N/kg) } & \multicolumn{3}{c|}{ Jump (N/kg) } & \multicolumn{3}{c|}{ Increase (\%) } \\
\cline { 2 - 11 } & Fy & Fz & Norm & Fy & Fz & Norm & Fy & Fz & Norm \\
\hline Shoulder & -21.64 & 1.9 & 21.7 & -47.4 & -2.9 & 47.5 & 119.0 & -252.6 & 118.6 \\
\hline Elbow & -37.7 & -10.9 & 39.2 & -73.1 & -12.8 & 74.2 & 93.9 & 17.4 & 89.1 \\
\hline Wrist & -50.2 & 4.73 & 50.4 & -76.7 & 16.8 & 78.5 & 52.8 & 255.2 & 55.7 \\
\hline MCP & -33.4 & 10.1 & 34.9 & -58.3 & 8.63 & 58.9 & 74.6 & -14.6 & 68.9 \\
\hline P2-P1 & -28.1 & 17.8 & 33.3 & -40.8 & 42.1 & 58.6 & 45.2 & 136.5 & 76.2 \\
\hline
\end{tabular}


Table 4. Comparison of values at trotting and jumping for the net joint moment and the joint reaction forces. The increase between trot and jumping is given in $\%$. 


\section{List of figures}

Fig. 1 Right forelimb equipped with the markers followed by Kinovea software.

Fig. 2 Top view of the running experiment.

Fig. 3 Procedure of correction of the trajectories.

Fig. 4 Methodology to find the joint local frames. Examples of the shoulder and the elbow.

Fig. 5 Reconstruction of the entire forelimb on CATIA V5 and construction of the OpenSim model from the Biosphera Software (without the wrapping surfaces).

Fig. 6 Solutions for adjusting the model.

Fig. 7 Trajectories of the markers before and after applying the solidification procedure.

Fig. 8 Joints angular variations across time.

Fig. 9 Ground reaction forces measured at trot.

Fig. 10 Reserves at trotting and jumping.

Fig. 11 Comparison of the vertical joint loading results for the trotting parameters (table 3 ) and the jumping parameters (table 4).

Fig. 12 Results of joint loading for trotting and jumping.

Fig. 13 Representation of the joint reaction forces in the right forelimb of a horse at trotting and at jumping a $1 \mathrm{~m}$ fence. 


\section{List of tables}

Table 1. Maximal reserves values at trot and jumping for the initial and final models

Table 2. Changes applied to the muscles to reduce the reserves at trot

Table 3. Changes applied to the muscles to reduce the reserves at jumping

Table 4. Comparison of values at trotting and jumping for the net joint moment and the joint reaction forces 